\title{
Differential effects of MTSS1 on invasion and proliferation in subtypes of non-small cell lung cancer cells
}

\author{
DONG-JIN LING ${ }^{1,2^{*}}$, ZHONG-SHU CHEN ${ }^{1,2^{*}}$, QIAN-DE LIAO $^{3}$, \\ JIAN-XIONG FENG ${ }^{2}$, XUE-YU ZHANG ${ }^{2}$ and TA-YAO YIN ${ }^{4}$
}

\begin{abstract}
${ }^{1}$ Ministry of Health Hepatobiliary and Enteric Surgery Center, Xiangya School of Medicine, Central South University, Changsha, Hunan 410078; ${ }^{2}$ Department of Thoracic Surgery, Jiangxi Provincial Chest Hospital, Nanchang, Jiangxi 330006;

${ }^{3}$ Department of Orthopaedics, Xiangya Hospital, Central South University, Changsha, Hunan 410078;

${ }^{4}$ Endoscopy Center, Jiangxi Provincial Chest Hospital, Nanchang, Jiangxi 330006, P.R. China
\end{abstract}

Received February 10, 2015; Accepted April 5, 2016

DOI: $10.3892 /$ etm.2016.3382

\begin{abstract}
Non-small cell lung cancer (NSCLC) accounts for $>80 \%$ of all cases of lung cancer and can be divided into lung adenocarcinoma (LAC), large-cell carcinoma (LCC), and squamous cell carcinoma (SCC). Accumulating evidence suggests that MTSS1, which is a newly discovered protein associated with tumor progression and metastasis, may have differential roles in cancer malignancy. As it has been demonstrated that MTSS1 is overexpressed in NSCLC and may be an independent prognostic factor in patients with SCC, the present study explored the differential roles of MTSS1 in the invasion and proliferation of different subtypes of NSCLC. Stable overexpression and knockdown of MTSS1 was performed in human NSCLC H920 (LAC), H1581 (LCC) and SW900 cell lines (SCC), and western blot, cell invasion, proliferation and FAK activity analyses were used to investigate the effects. Overexpression of MTSS1 enhanced the invasion and proliferation abilities of H920 and H1581 cells, and these effects were abolished by treatment with selective FAK inhibitor 14, which did not affect the expression of MTSS1. Notably, overexpression of MTSS1 inhibited invasion and proliferation in SW900 cells, and this effect was enhanced by the selective FAK inhibitor. Knockdown of MTSS1 decreased the invasion and proliferation abilities of H920 and H1581 cells, whereas knockdown increased invasion and proliferation in SW900 cells. Furthermore, while overexpression of MTSS1 induced FAK phosphorylation and activity in H920 and H1581 cells, MTSS1 overexpression inhibited FAK
\end{abstract}

Correspondence to: Dr Qian-De Liao, Department of Orthopaedics, Xiangya Hospital, Central South University, 87 Xiangya Road, Changsha, Hunan 410078, P.R. China

E-mail: liao.daguang@yahoo.com

*Contributed equally

Key words: metastasis suppressor 1, non-small cell lung cancer, lung adenocarcinoma, large-cell carcinoma, squamous cell carcinoma, invasion, proliferation, focal adhesion kinase phosphorylation/activity in SW900 cells. Knockdown of MTSS1 decreased FAK phosphorylation/activity in H920 and H1581 cells, whereas knockdown increased these processes in SW900 cells. To the best of our knowledge, the present study was the first to demonstrate that MTSS1 has differential roles in various subtypes of NSCLC, acting via a FAK-dependent mechanism. The results indicated that MTSS1 may enhance invasion and proliferation in LAC and LCC cells, whereas MTS11 inhibits these processes in SCC cells. These findings provide novel insight into the functional role of MTSS1 in cancer and may help elucidate therapeutic strategies for the treatment of various types of cancer.

\section{Introduction}

Lung cancer remains the leading cause of death from cancer worldwide, with $\sim 1.4$ million deaths each year (1). Non-small cell lung cancer (NSCLC), which is the predominant form of the disease, accounts for $>80 \%$ of all cases of lung cancer (2). There are three types of NSCLC: Lung adenocarcinoma (LAC), large-cell carcinoma (LCC) and squamous cell carcinoma (SCC) (3). Despite great advances in surgery, chemotherapy and radiotherapy, the long-term survival of patients with NSCLC remains poor due to rapid growth and high rate of recurrence and metastasis $(4,5)$. Therefore, it is imperative that the precise molecular mechanisms underlying the invasion and metastasis of NSCLC are investigated.

Metastasis suppressor 1 (MTSS1), which is also known as MIM (missing-in-metastasis), was first identified as a potential metastasis suppressor gene in metastatic bladder carcinoma cell lines (6). Accumulating evidence indicates that MTSS1 is a multifunctional molecular effector with an important role in cancer development, carcinogenesis and metastasis (7-9). As a scaffold protein, MTSS1 interacts with various partners to regulate actin dynamics $(8,9)$ and is also involved in the transcription of effector genes of the sonic hedgehog (Shh) signaling pathway (10). Whereas the expression of MTSS1 is reduced in prostate and breast cancers, it is upregulated in hepatocellular carcinoma (11). Furthermore, although MTSS1 reportedly inhibits the invasion and proliferation of breast, colorectal and prostate cancer cells (12-14), it has been demonstrated to be driver 
of metastasis in a subset of melanomas (15). Therefore, MTSS1 may have differential roles in cancer malignancy, depending on tissue/cell specificity. A previous study has demonstrated that MTSS1 is significantly overexpressed in NSCLC, as compared with normal lung tissue; among the subtypes of NSCLC, which include LAC, LCC and SCC, a significant correlation was detected between decreased MTSS1 expression and poor prognosis in patients with SCC (3).

To the best of our knowledge, the present study was the first to explore the differential roles of MTSS1 in the invasion and proliferation of the different subtypes of NSCLC, which may provide novel insight into the functional role of MTSS1 in cancer and may help elucidate therapeutic strategies for the treatment of various types of cancer.

\section{Materials and methods}

Cell lines and reagents. Human NSCLC cell lines H920 (LAC) (CRL-5850), H1581 (LCC) (CRL-5878), and SW900 (SCC) (HTB-59) cell lines were purchased from the American Tissue Culture Collection (Manassas, VA, USA) and cultured in Dulbecco's modified Eagle's medium (DMEM) supplemented with $10 \%$ heat-inactivated fetal bovine serum (both Thermo Fisher Scientific, Inc., Waltham, MA, USA) and $100 \mathrm{U} / \mathrm{ml}$ penicillin-streptomycin (Sigma-Aldrich, Beijing, China) at $37^{\circ} \mathrm{C}$ in an incubator with a humidified atmosphere composed of $95 \%$ air and 5\% $\mathrm{CO}_{2}$. Human MTSS1 cDNA clone (SC114849) was purchased from Origene (Beijing, China) and the full length MTSS1 cDNA sequence was subcloned into the pcDNA 3.1 plasmid. QCM ECMatrix 24-well $(8 \mu \mathrm{M})$ Fluorimetric Cell Invasion Assay kit (ECM554) was purchased from Chemicon (EMDMillipore,Billerica,CA,USA).Methylthiazoletetrazolium (MTT) Cell Proliferation Assay kit (30-1010 K) was purchased from ATCC. Universal Tyrosine Kinase Assay kit (MK410) was purchased from Takara Biotechnology Co., Ltd., (Beijing, China). Lipofectamine 2000 transfection reagent was purchased from Thermo Fisher Scientific, Inc. Human MTSS1 shRNA (sc-77651-V) lentiviral particles, control shRNA lentiviral particles-A (sc-108080), and mouse anti-human monoclonal MTSS1 (SS-3; sc-101204), mouse anti-human monoclonal focal adhesion kinase (FAK) (B-8; sc-271195), goat anti-human polyclonal phosphorylated FAK (Tyr576/577) (sc-21831) and mouse anti-human monoclonal anti-glyceraldehyde-3-phosphate dehydrogenase (GAPDH) (A-3; sc-137179) antibodies were purchased from Santa Cruz Biotechnology, Inc., (Santa Cruz, CA, USA). Puromycin, G418 and selective FAK inhibitor 14 were purchased from Sigma-Aldrich.

Transfection and lentiviral transduction. MTSS1 expression vector was transfected into cells using Lipofectamine 2000 transfection reagent, according to the manufacturer's protocol. Pools of stable transfectants were generated via selection with G418 $(700 \mu \mathrm{g} / \mathrm{ml})$, according to the manufacturer's protocol. Lentiviral transduction of the MTSS1 shRNA was performed and pools of stable transductants were generated via selection with puromycin (5 $\mu \mathrm{g} / \mathrm{ml}$; Sigma-Aldrich), according to the manufacturer's protocol.

Western blot analysis. Cells was lysed with a hypotonic buffer containing $2 \%$ Nonidet-P and a protease inhibitor cocktail
(Sigma-Aldrich) by sonication three times for $3 \mathrm{sec}$ on ice. Following centrifugation at $2,000 \mathrm{xg}$ for $15 \mathrm{~min}$ at $4^{\circ} \mathrm{C}$, the supernatant obtained was obtained and used for the determination of protein concentration via the Coomassie blue method and for subsequent steps. Equal amount of proteins $(2 \mathrm{mg})$ from each sample were separated by $10 \%$ SDS-polyacrylamide gel electrophoresis and blotted onto a polyvinylidene difluoride microporous membrane (EMD Millipore, Billerica, MA, USA). Membranes were blocked with 5\% skimmed milk powder in Tris-buffered saline and Tween-20. Then, membranes were incubated for $1 \mathrm{~h}$ with a 1:1,000 dilution of the following primary antibodies: Mouse anti-human monoclonal MTSS1 (SS-3; cat. no. sc-101204); mouse anti-human monoclonal focal adhesion kinase (FAK; B-8; cat. no. sc-271195); goat anti-human polyclonal phosphorylated FAK (Tyr576/577; cat. no sc-21831); and mouse anti-human monoclonal anti-GAPDH (A-3; cat. no. sc-137179) purchased from Santa Cruz Biotechnology, Inc.. Then, following washing with Tris-buffered saline with Tween 20, membranes were incubated with horseradish peroxidase-conjugated bovine anti-mouse (cat. no. sc-2371) or bovine anti-goat (cat. no. sc-2350) secondary antibodies purchased from Santa Cruz Biotechnology, Inc. (1:5,000) for $1 \mathrm{~h}$. An enhanced chemiluminescence kit (GE Healthcare, Shanghai, China) was used to reveal the peroxidase. Three independent experiments were performed. Western blot expression levels were quantitated using ImageJ software version 1.42 (National Institutes of Health, Bethesda, MD, USA).

Cell invasion assay. An QCM ECMatrix 24-well $(8 \mu \mathrm{M})$ Fluorimetric Cell Invasion Assay kit (Chemicon; EMD Millipore) was used to perform In vitro cell invasion assays, according to the manufacturer's protocol $(16,17)$. An insert polycarbonate membrane (pore size, $8 \mu \mathrm{M}$ ) was used. The insert in the invasion kit was coated with a thin layer of ECMatrix. Cells were seeded in the insert (upper chamber) at a density of $5 \times 10^{4}$ cells/well in serum-free DMEM. A total of $600 \mu \mathrm{l}$ complete medium supplemented with $10 \%$ fetal bovine serum was added to the lower chamber. Following 24-h incubation, invading cell numbers were determined via a fluorescent cell dose curve plotted using GraphPad Prism version 5.0 (GraphPad Software, Inc., La Jolla, CA, USA), according to the manufacturer's protocol. Three independent experiments were performed in duplicate.

MTT cell proliferation assay. An MTT Cell Proliferation Assay kit was used to determine in vitro cell proliferation, according to the manufacturer's protocol. Briefly, cells were cultured at a density of $15 \times 10^{3}$ cells/well in 96-well tissue culture plates and incubated at $37^{\circ} \mathrm{C}$ for $48 \mathrm{~h}$. At the end of the culture period, cells were washed with phosphate-buffered saline, and MTT reagents were added according to the manufacturer's protocol. Absorbance was measured at $570 \mathrm{~nm}$ using an ELISA plate reader. Three independent experiments were performed in triplicate.

FAK activity assay. A nonradioactive isotope solid-phase ELISA kit, which used the poly (Glu, Tyr) as a substrate (Universal Tyrosine Kinase Assay kit; Takara Biotechnology Co., Ltd.), was used to measure the kinase activity of FAK. FAK was purified from cells by immunoprecipitation with a mouse 

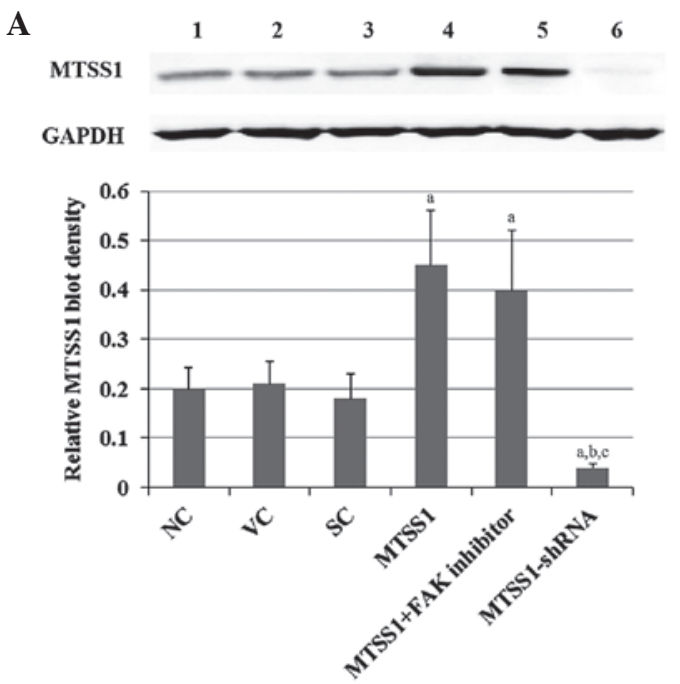

B
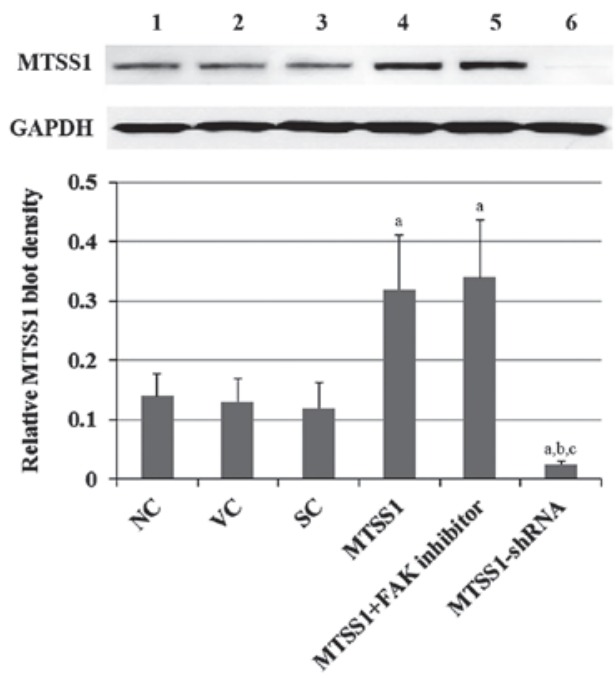
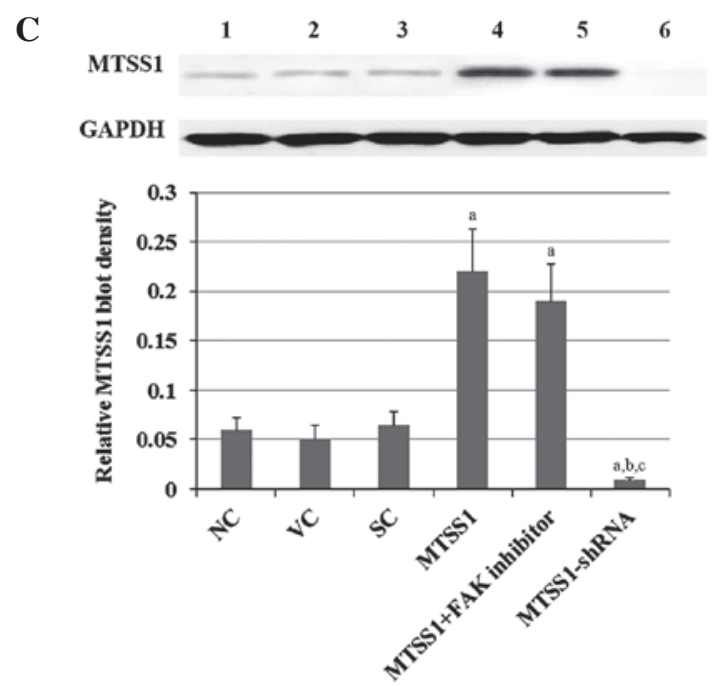

Figure 1. Protein levels of MTSS1 in subtypes of NSCLC cells, including (A) H920 lung adenocarcinoma, (B) H1581 large-cell carcinoma and (C) SW900 squamous cell carcinoma human NSCLC cells. Protein levels of MTSS1 were determined via western blot analyses in NC cells (lane 1), VC cells stably transfected with the empty pcDNA3.1 vector (lane 2), cells stably transduced with SC shRNA (lane 3), cells stably transfected with MTSS1 (lane 4), cells stably transfected with MTSS1 and treated with $50 \mu \mathrm{M}$ FAK inhibitor 14 for $24 \mathrm{~h}$ (lane 5), and cells stably transduced with MTSS1-shRNA (lane 6). GAPDH blotting was used as a loading control. MTSS1 blot densities were normalized against GAPDH to obtain a relative blot density. Data are expressed as the mean + standard deviation from three independent experiments. ${ }^{a} \mathrm{P}<0.05$ vs. the control groups (NC, VC and SC); ${ }^{\text {b }}<0.05$ vs. the $\mathrm{MTSS} 1$ group; ${ }^{\mathrm{c}} \mathrm{P}<0.05$ vs. the MTSS1 + FAK inhibitor group. MTSS1, metastasis suppressor 1; NSCLC, non-small cell lung cancer; FAK, focal adhesion kinase; NC, normal control; VC, vector control; SC, scramble control.

anti-human monoclonal FAK antibody (cat. no. sc-271195; Santa Cruz Biotechnology, Inc.). Briefly, $10 \mu \mathrm{g}$ antibody was pre-adsorbed on protein A Sepharose beads (Thermo Fisher Scientific, Inc.) in the presence of $2 \mathrm{mg} / \mathrm{ml}$ bovine serum albumin (Thermo Fisher Scientific, Inc.), and the beads were incubated with $1 \mathrm{ml}$ lysate for $2 \mathrm{~h}$ at $4^{\circ} \mathrm{C}$. Beads were washed 4 times with $1 \mathrm{ml}$ lysis buffer (Thermo Fisher Scientific, Inc.) and incubated for $5 \mathrm{~min}$ at room temperature with $40 \mu \mathrm{l}$ high salt radioimmunoprecipitation assay buffer $(50 \mathrm{mM}$ Tris, $250 \mathrm{mM}$ $\mathrm{NaCl}, 1 \% \mathrm{NP}-40,0.5 \%$ DOC, $0.1 \%$ SDS; $\mathrm{pH} 7.5$ ) in order to elute co-immunoprecipitated FAK. Immunoprecipitates were subjected to the in vitro kinase assay as per the manufacturer's protocol. Three independent experiments were performed in duplicate.

Statistical analysis. Statistical analyses were performed with SPSS 10.0 for Windows (SPSS, Inc., Chicago, IL, USA). All data values were expressed as the mean \pm standard deviation. Comparisons of means among multiple groups were performed with one-way analysis of variance, followed by post-hoc pairwise comparisons using Tukey's tests. $\mathrm{P}<0.05$ was considered to indicate a statistically significant difference.

\section{Results}

MTSS1 is stably overexpressed and knocked down in subtypes of NSCLC. In order to determine the functional role of MTSS1 in three subtypes of NSCLC, MTSS1 was stably overexpressed and knocked down in human H920 (LAC), H1581 (LCC) and SW900 (SCC) NSCLC cell lines. As detected by western blot analyses, H920 (Fig. 1A) and H1581 (Fig. 1B) cells exhibited higher constitutive expression levels of MTSS1, as compared with SW900 cells (Fig. 1C). Compared with the controls, MTSS1 expression levels were increased 2-fold in H920 and H1581 
A

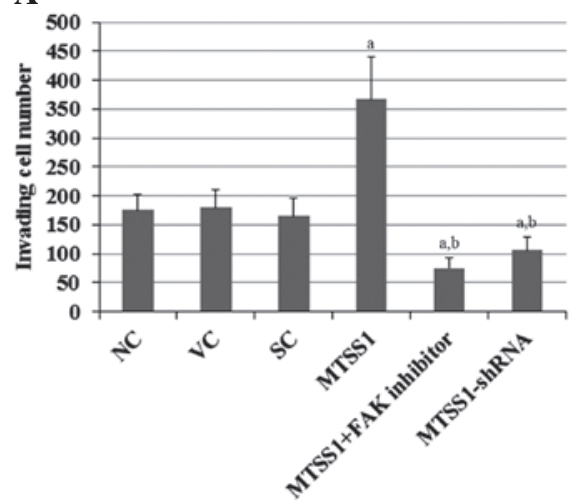

B

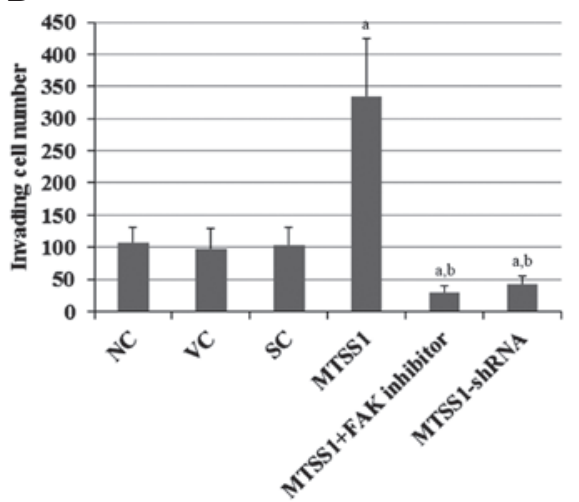

C

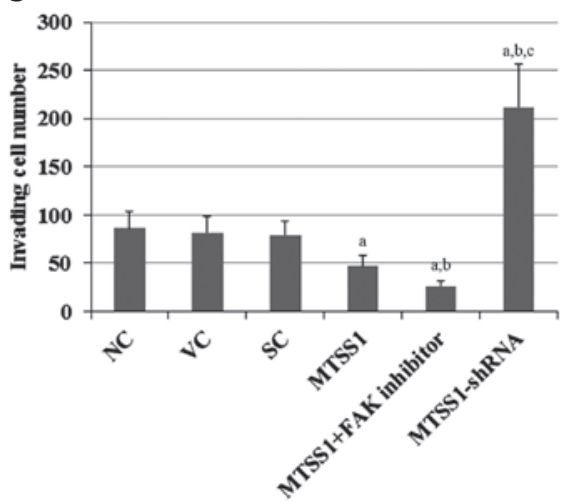

Figure 2. Effects of MTSS1 on cell invasion in various subtypes of NSCLC cells. In vitro cell invasion assays were performed in (A) H920 lung adenocarcinoma, (B) H1581 large-cell carcinoma and (C) SW900 squamous cell carcinoma human NSCLC cells. Following 24-h incubation, invading cell numbers were determined by running a fluorescent cell dose curve in NC cells, VC cells stably transfected with the empty pcDNA3.1 vector, SC cells stably transduced with scramble control shRNA, cells stably transfected with MTSS1, cells stably transfected with MTSS1 and treated with $50 \mu$ M FAK inhibitor 14 for 24 h, and cells stably transduced with MTSS1-shRNA. Data are expressed as the mean + standard deviation from three independent experiments. ${ }^{a} \mathrm{P}<0.05$ vs. the control groups (NC, VC and SC); ${ }^{\mathrm{b}} \mathrm{P}<0.05$ vs. the MTSS1 group; ${ }^{\mathrm{C}} \mathrm{P}<0.05$ vs. the MTSS1 + FAK inhibitor group. MTSS1, metastasis suppressor 1 ; NSCLC, non-small cell lung cancer; FAK, focal adhesion kinase; NC, normal control; VC, vector control; SC, scramble control.
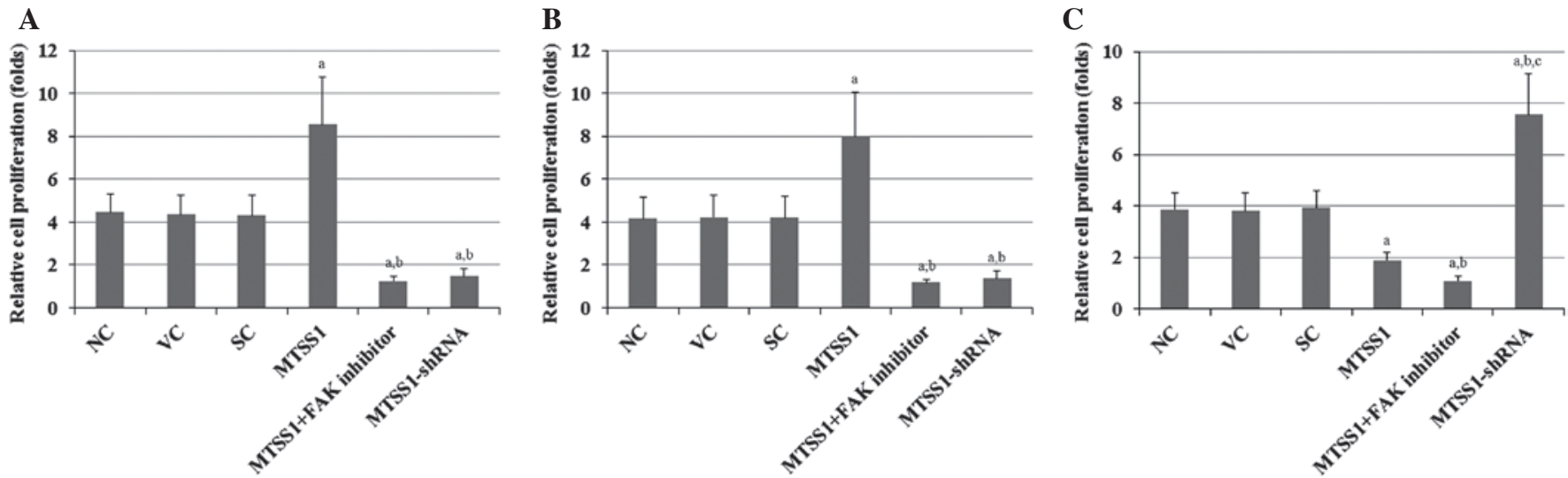

Figure 3. Effects of MTSS1 on cell proliferation in various subtypes of NSCLC cells, including (A) H920 lung adenocarcinoma, (B) H1581 large-cell carcinoma and (C) SW900 squamous cell carcinoma human NSCLC cells. MTT cell proliferation assays were performed for $48 \mathrm{~h}$ in NC cells, VC cells stably transfected with the empty pcDNA3.1 vector, SC cells stably transduced with scramble control shRNA, cells stably transfected with MTSS1, cells stably transfected with MTSS1 and treated with $50 \mu \mathrm{M}$ FAK inhibitor 14 for $48 \mathrm{~h}$, and cells stably transduced with MTSS1-shRNA. Cell proliferation at $48 \mathrm{~h}$ was expressed as fold change relative to the $\mathrm{NC}$ group at $0 \mathrm{~h}$ (designated as 1). Data are expressed as the mean + standard deviation from three independent experiments. ${ }^{a} \mathrm{P}<0.05$ vs. the control groups (NC, VC and SC); ${ }^{\circ} \mathrm{P}<0.05$ vs. the MTSS1 group; ${ }^{\mathrm{C}} \mathrm{P}<0.05$ vs. the MTSS1 + FAK inhibitor group. MTSS1, metastasis suppressor 1; NSCLC, non-small cell lung cancer; FAK, focal adhesion kinase; NC, normal control; VC, vector control; SC, scramble control.

cells and 3 -fold in SW900 cells (Fig. 1). Lentiviral transduction of shRNA knocked down the endogenous level of MTSS1 by 280\% in H920 and H1581 cells and by 85\% in SW900 cells. Treatment with selective FAK inhibitor 14 induced no significant alterations in the expression levels of MTSS1, as compared with the MTSS1 group, in all three cell lines (Fig. 1). The results indicate that MTSS1 is successfully overexpressed and knocked down in NSCLC cells, respectively.

MTSS1 exerts differential effects on invasion in subtypes of NSCLC. In order to determine the effects of MTSS1 on cell invasion in three subtypes of NSCLC cells, in vitro cell invasion assays were performed in H920, H1581 and SW900 cells. Compared with the controls, overexpression of MTSS1 increased the invading cell number of H920 and H1581 cells by 2- and $\sim 3$-fold, respectively (Figs. 2A and B). Notably, this effect was abolished by treatment with selective FAK inhibitor 14. In contrast, overexpression of MTSS1 decreased the invading cell number by $\sim 50 \%$ in SW900 cells, and treatment with selective FAK inhibitor 14 enhanced this effect (Fig. 2C). Knockdown of MTSS1 decreased the invading cell number by 40 and $60 \%$ in H920 and H1581 cells, respectively; whereas it increased the invading cell number of SW900 cells 2.5 -fold (Fig. 2). The results indicate that MTSS1 has differential effects on the invasiveness of different subtypes of NSCLC.

MTSS1 exerts differential effects on proliferation in subtypes of NSCLC. In order to determine the effects of MTSS1 on cell proliferation in three subtypes of NSCLC cells, MTT in vitro cell proliferation assays were performed in H920, H1581 and SW900 cells. Compared with the controls, overexpression of MTSS1 increased the proliferation of H920 and H1581 cells 2-fold, and this effect was abolished by treatment with selective FAK inhibitor 14 (Figs. 3A and B). In contrast, overexpression 

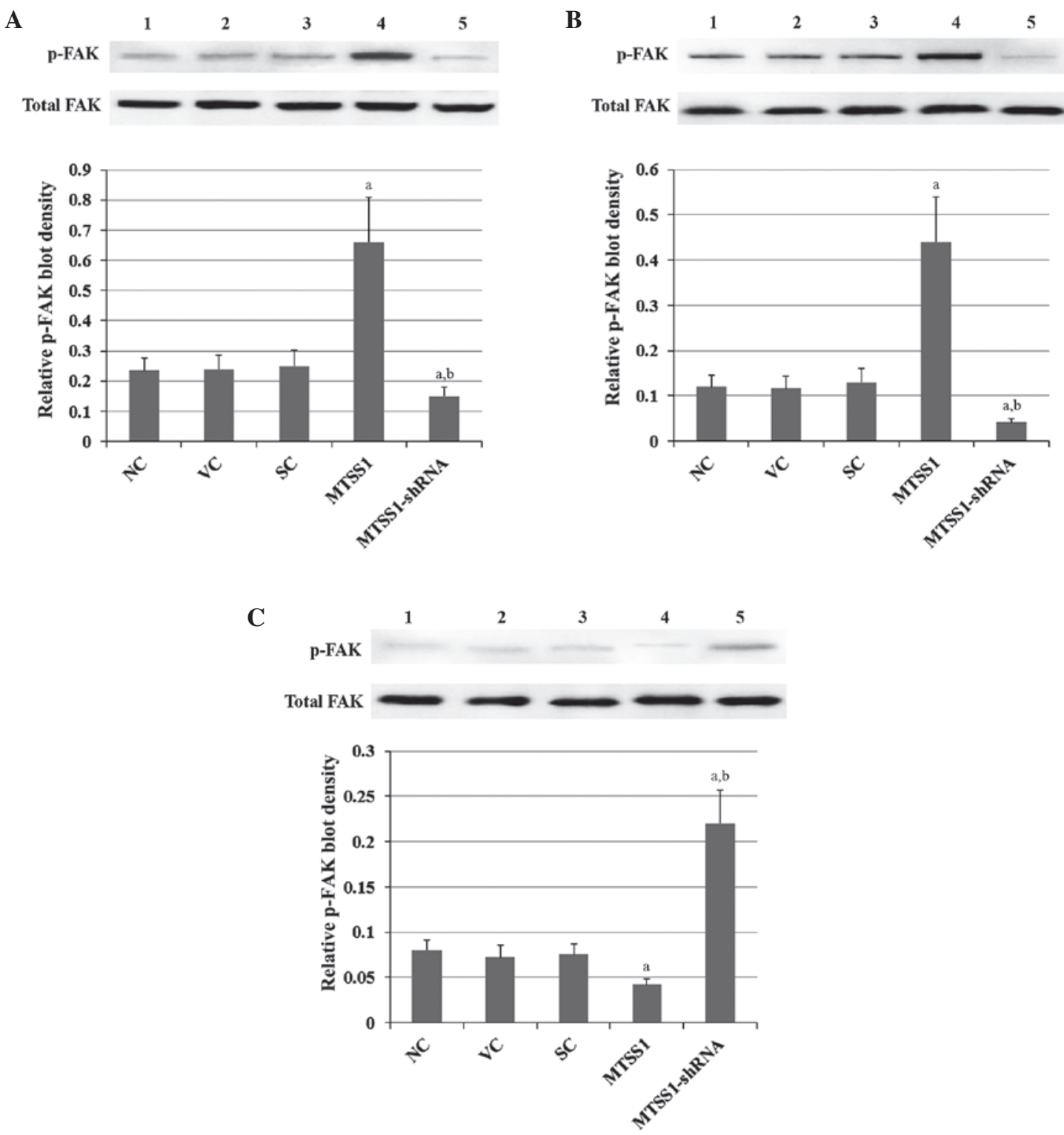

Figure 4. Effects of MTSS1 on the tyrosine 576/577 phosphorylation of FAK in various subtypes of NSCLC cells, including (A) H920 lung adenocarcinoma, (B) H1581 large-cell carcinoma and (C) SW900 squamous cell carcinoma human NSCLC cells. Levels of total FAK and p-FAK were determined by western blot analyses in NC cells (lane 1), VC cells stably transfected with the empty pcDNA3.1 vector (lane 2), SC cells stably transduced with scramble control shRNA (lane 3), cells stably transfected with MTSS1 (lane 4) and cells stably transduced with MTSS1-shRNA (lane 5). p-FAK blot density was normalized against total FAK to obtain a relative p-FAK blot density. Data are expressed as the mean + standard deviation from three independent experiments. ${ }^{\text {a }}<0.05$ vs the control groups (NC, VC and SC); ${ }^{\text {P }}<0.05$ vs. the MTSS1 group. MTSS1, metastasis suppressor 1; NSCLC, non-small cell lung cancer; FAK, focal adhesion kinase; p-, phosphorylated; NC, normal control; VC, vector control; SC, scramble control.

of MTSS1 decreased cell proliferation by 50\% in SW900 cells, and this effect was enhanced by treatment with selective FAK inhibitor 14 (Fig. 3C). Knockdown of MTSS1 decreased the proliferation of $\mathrm{H} 920$ and $\mathrm{H} 1581$ cells by $~ 65 \%$, whereas it increased the proliferation of SW900 cells $\sim 2$-fold (Fig. 3). The results indicate that MTSS1 has differential effects on the proliferation of different subtypes of NSCLC.

MTSS1 exerts differential effects on the phosphorylation of $F A K$ and FAK activity in subtypes of NSCLC. As the findings of the present so far suggested that MTSS1 promoted and inhibited cell invasion and proliferation in three subtypes of human NSCLC cells through a FAK-dependent mechanism, the effects of MTSS1 on the phosphorylation of FAK and FAK activity in H920, H1581 and SW900 cells were investigated. Since phosphorylation of FAK on tyrosine (Tyr) 576 and 577 within the FAK catalytic domain is required for the full enzymatic activity of FAK $(18,19)$, Tyr576/577-phosphorylated FAK protein levels were measured in the cells. Compared with the controls, overexpression and knockdown of MTSS1 showed no significant effects on total FAK protein levels in all three cell lines (Fig. 4). In contrast, overexpression of MTSS1 increased FAK phosphorylation at Tyr576/577 by $~ 3$-fold in H920 and H1581 cells (Figs. 4A and B), whereas MTSS1 overexpression decreased Tyr576/577-phosphorylated FAK protein levels by $\sim 50 \%$ in SW900 cells (Fig. 4C). Knockdown of MTSS1 decreased Tyr576/577 phosphorylation in FAK by $\sim 60$ and $65 \%$ in H920 and H1581 cells, respectively 
A

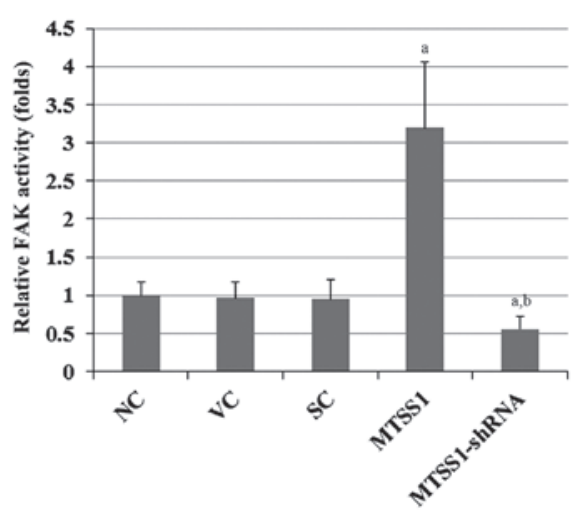

B

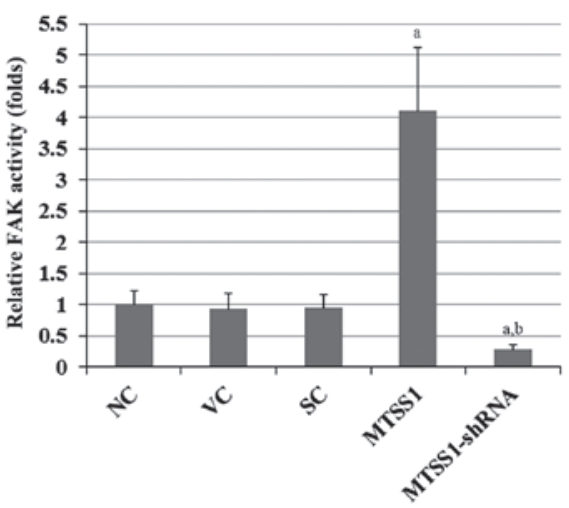

C

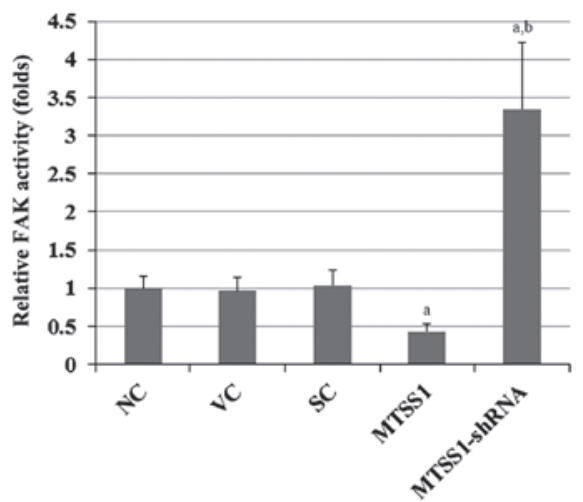

Figure 5. Effect of MTSS1 on FAK activity in various subtypes of NSCLC cells, including (A) H920 lung adenocarcinoma, (B) H1581 large-cell carcinoma and (C) SW900 squamous cell carcinoma human NSCLC cells. FAK activity was determined using a Universal Tyrosine Kinase Assay kit in NC cells, VC cells stably transfected with the empty pcDNA3.1 vector, SC cells stably transduced with scramble control shRNA, cells stably transfected with MTSS1, and cells stably transduced with MTSS1-shRNA. FAK activity is presented as fold change, relative to the NC group (designated as 1). Data are expressed as the mean + standard deviation from three independent experiments. ${ }^{a} \mathrm{P}<0.05$ vs.the control groups (NC, VC and SC); ${ }^{b} \mathrm{P}<0.05$ vs. the MTSS1 group. MTSS1, metastasis suppressor 1; NSCLC, non-small cell lung cancer; FAK, focal adhesion kinase; NC, normal control; VC, vector control; SC, scramble control.

(Figs 5A and B), whereas MTSS1 knockdown increased Tyr576/577 phosphorylation in FAK by 3-fold in SW900 cells (Fig. 4C). Similar trends in FAK activity were observed in the cell lines (Fig. 5). The results indicate that MTSS1 has differential effects on FAK activity in different subtypes of NSCLC.

\section{Discussion}

Decreased expression of MTSS1, which is a newly discovered protein involved in tumor progression and metastasis (3), has been associated with poor survival rate in patients with aggressive forms of breast, colorectal and prostate cancer (12-14). Conversely, elevated expression of MTSS1 has been associated with enhanced invasion and proliferation in melanoma cells and characterizes a subgroup of primary melanomas with unfavorable prognosis (15). Furthermore, a recent study has demonstrated that MTSS1 is overexpressed in NSCLC and may be an independent prognostic factor for patients with SCC (3). To the best of our knowledge the present study was the first to outline the differential role of MTSS1 in invasion and proliferation in three subtypes of NSCLC.

NSCLC consists of LAC, LCC and SCC (3). In the present study, H920 (LAC), H1581 (LCC) and SW900 (SCC) cell lines were used as representative cell models of the subtypes of NSCLC. Since all three cell lines were derived from stage-4 NSCLC, they possess similar pathological characteristics. Stable overexpression and knockdown of MTSS1 was performed in the cell lines in order to explore the functional roles of MTSS1 in NSCLC cell invasion and proliferation. Notably, the results of the present study demonstrated that, while MTSS1 overexpression inhibited invasion and proliferation in SCC cells, it enhanced invasion and proliferation in LAC and LCC cells. When together with previous reports that MTSS1 respectively enhances and inhibits tumor cell invasion and proliferation in different types of cancer (12-15), these findings suggest that MTSS1 has a dual functional role of oncogene and tumor suppressor in cancer, depending on tissue/cell specificity. Previous studies have described various similar proteins with dual roles in cancer malignancy $(19,20)$. For example, while Annexin A5 reportedly promotes tumorigenesis and progression in hepatocarcinoma, breast cancer, colorectal cancer, pancreatic cancer, bladder cancer and prostate cancer (19), it has also been demonstrated to inhibit the malignancy of thyroid cancer (19). Moreover, high expression of TWIST, which is a transcription factor belonging to the basic helix-loop-helix family of proteins (20), has been associated with the initial phase of metastatic progression in gastric and breast cancers, and its overexpression is correlated with disease progression and a poor clinical outcome in patients with osteosarcoma (20). As MTSS1 is associated with the invasion and proliferation of various types of cancers and it has been suggested that MTSS1 may serve as a prognostic indicator and/or therapeutic target for cancer $(12-15,21)$, identification of the differential roles of MTSS1 in the invasion and proliferation of various subtypes of NSCLC may provide novel insights into the functional role of MTSS1 in cancer.

As a scaffold protein that interacts with numerous molecules to regulate actin dynamics, MTSS1 has an important role in carcinogenesis and metastasis (7), and is also associated with the Shh signaling pathway (10). In the present study, the effects of MTSS1 on the invasion and proliferation of NSCLC cells were investigated in the presence of a selective FAK inhibitor, which did not affect the expression of MTSS1. The results of the present study suggested that MTSS1 may regulate NSCLC cell invasion and proliferation through a FAK-dependent mechanism, regardless of whether a stimulatory or inhibitory effect is evoked. It is well-established that focal adhesion complexes are capable of controlling cell movement by associating with the actin cytoskeleton (22); therefore, dynamic regulation of focal adhesion complexes and reorganization of the associated actin cytoskeleton are crucial determinants of tumor cell invasion (22). As a critical factor involved in the mechanism of focal adhesion, FAK may function as a major downstream mediator of the regulatory effects of MTSS1 on actin dynamics. The results of the present study 
demonstrated that, while MTSS1 overexpression induced FAK phosphorylation/activity in LAC and LCC cells, it inhibited the same processes in SCC cells. Therefore, the mechanism by which MTSS1 exerts differential effects on the FAK phosphorylation/activity in different subtypes of NSCLC may be dependent on specific intracellular partners that interact with MTSS1 in specific cancer cell types. Future studies are required in order to elucidate these underlying mechanisms.

In conclusion, the present study demonstrated that MTSS1 has differential roles in various subtypes of NSCLC. Acting via a FAK-dependent mechanism, MTSS1 enhances the invasion and proliferation of LAC and LCC cells, whereas it inhibits the invasion and proliferation of SCC cells. These findings provide novel insight into the functional role of MTSS1 in cancer and may help elucidate therapeutic strategies for the treatment of various types of cancer.

\section{References}

1. Ramalingam SS, Owonikoko TK and Khuri FR: Lung cancer: New biological insights and recent therapeutic advances. CA Cancer J Clin 61: 91-112, 2011

2. Chang A: Chemotherapy, chemoresistance and the changing treatment landscape for NSCLC. Lung Cancer 71: 3-10, 2011.

3. Kayser G, Csanadi A, Kakanou S, Prasse A, Kassem A, Stickeler E, Passlick B and Zur Hausen A: Downregulation of MTSS1 expression is an independent prognosticator in squamous cell carcinoma of the lung. Br J Cancer 112: 866-873, 2015.

4. Peters S, Adjei AA, Gridelli C, Reck M, Kerr K and Felip E; ESMO Guidelines Working Group: Metastatic non-small-cell lung cancer (NSCLC): ESMO clinical practice guidelines for diagnosis, treatment and follow-up. Ann Oncol 23 (Suppl 7): vii56-vii64, 2012.

5. Li H, Zhang Y, Zhang Y, Bai X, Peng Y and He P: TRIM31 is downregulated in non-small cell lung cancer and serves as a potential tumor suppressor. Tumour Biol 35: 5747-5752, 2014.

6. Lee Y, Macoskay JA, Korenchukx S and Pientay KJ: MIM, a potential metastasis suppressor gene in bladder cancer. Neoplasia 4: 291-294, 2002.

7. Xie F, Ye L, Ta M, Zhang L and Jiang WG: MTSS1: A multifunctional protein and its role in cancer invasion and metastasis. Front Biosci (Schol Ed) 3: 621-631, 2011.

8. Mattila PK, Salminen M, Yamashiro T and Lappalainen P: Mouse MIM, a tissue-specific regulator of cytoskeletal dynamics, interacts with ATP-actin monomers through its C-terminal WH2 domain. J Biol Chem 278: 8452-8459, 2003.
9. Mattila PK, Pykäläinen A, Saarikangas J, Paavilainen VO, Vihinen H, Jokitalo E and Lappalainen P: Missing-in-metastasis and IRSp53 deform PI $(4,5)$ P2-rich membranes by an inverse BAR domain-like mechanism. J Cell Biol 176: 953-964, 2007.

10. Callahan CA, Ofstad T, Horng L, Wang JK, Zhen HH, Coulombe PA and Oro AE: MIM/BEG4, a Sonic hedgehog-responsive gene that potentiates Gli-dependent transcription. Genes Dev 18: 2724-2729, 2004.

11. Ma S, Guan X, Lee TK and Chan KW: Clinicopathological significance of missing in metastasis B expression in hepatocellular carcinoma. Hum Pathol 38: 1201-1206, 2007.

12. Lei R, Tang J, Zhuang X, Deng R, Li G, Yu J, Liang Y, Xiao J, Wang HY, Yang Q and Hu G: Suppression of MIM by microRNA-182 activates RhoA and promotes breast cancer metastasis. Oncogene 33: 1287-1296, 2014.

13. Zhou W, Li X, Liu F, Xiao Z, He M, Shen S and Liu S: MiR-135a promotes growth and invasion of colorectal cancer via metastasis suppressor 1 in vitro. Acta Biochim Biophys Sin (Shanghai) 44: 838-846, 2012.

14. Mustafa N, Martin TA and Jiang WG: Metastasis tumour suppressor-1 and the aggressiveness of prostate cancer cells. Exp Ther Med 2: 157-162, 2011.

15. Mertz KD, Pathria G, Wagner C, Saarikangas J, Sboner A, Romanov J, Gschaider M, Lenz F, Neumann F, Schreiner W, et al: MTSS1 is a metastasis driver in a subset of human melanomas. Nat Commun 5: 3465, 2014.

16. Wang B, Feng P, Xiao Z and Ren EC: LIM and SH3 protein 1 (Lasp1) is a novel p53 transcriptional target involved in hepatocellular carcinoma. J Hepatol 50: 528-537, 2009.

17. Feng Y, Hu J, Ma J, Feng K, Zhang X, Yang S, Wang W, Zhang $\mathrm{J}$ and Zhang Y: RNAi-mediated silencing of VEGF-C inhibits non-small cell lung cancer progression by simultaneously down-regulating the CXCR4, CCR7, VEGFR-2 and VEGFR-3-dependent axes-induced ERK, p38 and AKT signalling pathways. Eur J Cancer 47: 2353-2363, 2011.

18. McLean GW, Carragher NO, Avizienyte E, Evans J, Brunton VG and Frame MC: The role of focal-adhesion kinase in cancer-a new therapeutic opportunity. Nat Rev Cancer 5: 505-515, 2005

19. Peng B, Guo C, Guan H, Liu S and Sun MZ: Annexin A5 as a potential marker in tumors. Clin Chim Acta 427: 42-48, 2014.

20. Zhou Y, Huang Z, Wu S, Zang X, Liu M and Shi J: miR-33a is up-regulated in chemoresistant osteosarcoma and promotes osteosarcoma cell resistance to cisplatin by down-regulating TWIST. J Exp Clin Cancer Res 33: 12, 2014.

21. Xie F, Ye L, Chen J, Wu N, Zhang Z, Yang Y, Zhang L and Jiang WG: The impact of Metastasis Suppressor-1, MTSS1, on oesophageal squamous cell carcinoma and its clinical significance. J Transl Med 9: 95, 2011.

22. Carragher NO and Frame MC: Focal adhesion and actin dynamics: A place where kinases and proteases meet to promote invasion. Trends Cell Biol 14: 241-249, 2004. 